\title{
LIE INNER IDEALS ARE NEARLY JORDAN INNER IDEALS
}

\author{
ANTONIO FERNÁNDEZ LÓPEZ \\ (Communicated by Kailash C. Misra) \\ Dedicated to Professor Georgia Benkart
}

\begin{abstract}
In this note we extend the Lie inner ideal structure of simple Artinian rings developed by Benkart to centrally closed prime algebras $A$. New Lie inner ideals, which we call nonstandard, occur when making this extension. A necessary and sufficient condition for $A$ to have a nonstandard inner ideal is the existence in $A$ of a zero square element which is not von Neumann regular. Our main tool is a theorem due to Martindale and Miers on the iterates of the derivations of prime rings.
\end{abstract}

\section{INTRODUCTION}

Inner ideals of Lie algebras are the analogues of one-sided ideals in associative rings and algebras. They are subspaces $B$ of a Lie algebra $L$ such that $[[B, L], B] \subseteq B$. An abelian inner ideal of $L$ is an inner ideal $B$ which is also an abelian subalgebra, i.e., $[B, B]=0$. Since their introduction over 30 years ago ([7], 3]), abelian inner ideals have proved to be a useful tool for classifying both finite-dimensional and infinite-dimensional simple Lie algebras. Premet ([18, [19]) has shown that every finite-dimensional simple Lie algebra over an algebraically closed field of characteristic not 2 or 3 must contain one-dimensional inner ideals. Moreover, it follows from ([3], 20]) (see also [4]) that when the field is algebraically closed of characteristic $p>5$, the classical Lie algebras (modular versions of the complex finite-dimensional simple Lie algebras) can be characterized as the finitedimensional simple Lie algebras satisfying the following two equivalent conditions:

(i) they are generated by one-dimensional inner ideals;

(ii) they are nondegenerate (that is, they have no nonzero absolute zero divisors, where by an absolute zero divisor (or sandwich element) we mean an element $x$ such that $[x,[x, L]]=0)$.

Further evidence of the usefulness of inner ideals comes from [12, where it is shown that an abelian inner ideal $B$ of finite length in an arbitrary nondegenerate Lie algebra $L$ over a commutative ring $\Phi$ such that 2 and 3 are invertible in $\Phi$ gives rise to a finite $\mathbb{Z}$-grading $L=L_{-n} \oplus \cdots \oplus L_{0} \oplus \cdots \oplus L_{n}$ with $B=L_{n}$. Zelmanov 23. described the simple Lie algebras over fields of characteristic 0 or $p>4 n+1$ with such gradings in terms of finite $\mathbb{Z}$-gradings of simple associative

Received by the editors November 17, 2011 and, in revised form, April 9, 2012.

2010 Mathematics Subject Classification. Primary 17B60, 17C50; Secondary 16N60.

Key words and phrases. Central closed prime algebra, Jordan-Lie inner ideal.

The author was supported in part by the MEC and Fondos FEDER, MTM2010-19482. 
rings with involution. A description of these associative rings and their gradings was later provided by Smirnov in [21, 22. As a result, any nondegenerate simple Lie algebra with a nonzero abelian inner ideal of finite length comes from a simple associative ring with a finite $\mathbb{Z}$-grading by taking the Lie commutator from the skew-symmetric elements of such a simple associative ring with involution or from the Tits-Kantor-Koecher construction of a Jordan algebra of a symmetric bilinear form, or it is of exceptional type $\mathrm{E}_{6}, \mathrm{E}_{7}, \mathrm{E}_{8}, \mathrm{~F}_{4}, \mathrm{G}_{2}$.

We also note that there is a strong connection between inner ideals of Lie algebras and inner ideals of Jordan systems (algebras and pairs, [14]) which has been developed in a series of articles during the last five years $([8]-[12,[5], 6])$. In particular, results from [12 enable us to adopt a Jordan approach based on the notion of a subquotient of an abelian inner ideal to obtain the desired Lie theoretic theorems.

Let $A$ be an associative algebra (not necessarily unital) over a ring of scalars $\Phi$. By a Lie inner ideal of $A$ we mean an abelian inner ideal $B$ of the Lie algebra $A^{-}$; i.e., $B$ is a $\Phi$-submodule of $A$ such that $[[B, A], B] \subseteq B$ and $[B, B]=0$. Benkart proved in [2, Corollary 3.13] that if $A$ is simple of characteristic not 2 or 3 , then any proper inner ideal of the Lie algebra $[A, A]$ is a Lie inner ideal of $A$ and described these inner ideals when $A$ is Artinian [2, Theorem 5.1].

Suppose now that $\frac{1}{2} \in \Phi$. By a Jordan inner ideal of $A$ we mean an inner ideal $V$ of the Jordan algebra $A^{+}$, i.e., $v A v \subseteq V$ for all $v \in V$. It is easy to see that if $V$ is a $\Phi$-submodule of $A$ such that $V V=0$, then $V$ is a Jordan inner ideal if and only if it is a Lie inner ideal. In this case, $V$ will be called a Jordan-Lie inner ideal. If $V$ is a Jordan-Lie inner ideal, then for any $\Phi$-module $\Omega$ of $Z(A), V+\Omega$ is a Lie inner ideal. Any Lie inner ideal of this form, $B=V+\Omega$, will be called standard.

In this note we study conditions under which a Lie inner ideal of a semiprime associative algebra is standard, give a construction of nonstandard inner ideals, and classify the Lie inner ideals of any centrally closed prime algebra of characteristic not 2 or 3 .

We also prove that if $B$ is a Lie inner ideal of $A$ such that its image $\bar{B}$ in the Lie algebra $A^{-} / Z(A)$ is von Neumann regular, in the Lie sense, then $B$ is standard. Then using the Jordan structure theory for Lie algebras developed in [10] and [12], we prove that the von Neumann regularity of $\bar{B}$ is guaranteed when it has finite length.

Since any simple algebra is centrally closed over its centroid, we derive from our previous results that any Lie inner ideal of a semiprime associative algebra $A$ coinciding with its socle is standard. In fact, we describe its Lie inner ideals and refine the description in the case where $A$ is Artinian. The reader is referred to [2, Theorem 5.1] and [9, Theorem 2.5] for related results.

\section{Associative algebras, Lie Algebras and Jordan systems}

Throughout this section, and unless otherwise specified, we will be dealing with (not necessarily unital) associative algebras $A$, with product $x y$; Lie algebras $L$, with $[x, y]$ denoting the Lie bracket and $\operatorname{ad}_{x}$ the adjoint map determined by $x$; Jordan pairs $V=\left(V^{+}, V^{-}\right)$, with triple products $\{x, y, z\}$, for $x, z \in V^{\sigma}, y \in V^{-\sigma}$, $\sigma= \pm$, and quadratic operators $Q_{x} y=\frac{1}{2}\{x, y, x\}$; and Jordan algebras $J$, with product $x \cdot y$, quadratic operator $U_{x} y=2 x \cdot(x \cdot y)-x^{2} \cdot y$ and triple product $\{x, y, z\}=U_{x+z} y-U_{x} y-U_{z} y$, over a ring of scalars $\Phi$ containing $\frac{1}{6}$. So Jordan 
pairs and Jordan algebras considered here are linear. Since any Jordan algebra can be regarded as a Jordan pair, any definition for Jordan pairs makes sense for Jordan algebras. The reader is referred to [14] as a general reference for Jordan pairs.

2.1. Any associative algebra $A$ gives rise to:

(i) a Lie algebra $A^{-}$, with Lie bracket $[x, y]=x y-y x$,

(ii) a Jordan pair $(A, A)$, with Jordan triple products given by $\{x, y, z\}=$ $x y z+z y x$

(iii) a Jordan algebra $A^{+}$, with Jordan product $x \cdot y=\frac{1}{2}(x y+y x)$, quadratic operator $U_{x} y=x y x$ and triple product $\{x, y, z\}=x y z+z y x$.

2.2. Given a Jordan pair $V=\left(V^{+}, V^{-}\right)$, an inner ideal of $V$ is any $\Phi$-submodule $B$ of $V^{\sigma}$ such that $\left\{B, V^{-\sigma}, B\right\} \subseteq B$. Similarly, an inner ideal of a Lie algebra $L$ is a $\Phi$-submodule $B$ of $L$ such that $[[B, L], B] \subseteq B$. An abelian inner ideal is an inner ideal $B$ which is also an abelian subalgebra, i.e., $[B, B]=0$.

2.3. Let $A$ be an associative algebra. An abelian inner ideal of $A^{-}$will be called a Lie inner ideal of $A$. Similarly, an inner ideal of the Jordan algebra $A^{+}$will be called a Jordan inner ideal of $A$.

Proposition 2.4. An ad-nilpotent element $x \in L$ of index of nilpotency at most 3 is called a Jordan element.

(i) Clearly, any element of an abelian inner ideal is a Jordan element. Conversely, by [3, 1.8], any Jordan element $b \in L$ yields the principal abelian inner ideal $\operatorname{ad}_{b}^{2} L$.

(ii) Any zero square element $x$ in an associative algebra $A$ is a Jordan element of the Lie algebra $A^{-}$. Indeed, $x^{2}=0$ implies $\operatorname{ad}_{x}^{2} y=-2 x y x$ for all $y \in A$, and hence $\operatorname{ad}_{x}^{3}=0$.

2.5. Recall that an element $x$ in an associative algebra $A$ is von Neumann regular if $x \in x A x$. Similarly, an element $x \in V^{\sigma}, \sigma= \pm$, in a Jordan pair $V$ is von Neumann regular if $x \in Q_{x} V^{-\sigma}$.

2.6. A Jordan element $e$ in a Lie algebra $L$ is called von Neumann regular if $e \in$ $\operatorname{ad}_{e}^{2} L$. It is easy to see that if $x$ is an element of an associative algebra such that $x^{2}=0$, then $x$ is von Neumann regular in the associative sense if and only if it is von Neumann regular in the Lie sense.

2.7. Let $V=\left(V^{+}, V^{-}\right)$be a Jordan pair. An element $x \in V^{\sigma}, \sigma= \pm$, is called an absolute zero divisor if $Q_{x} V^{-\sigma}=0$. A Jordan pair $V$ is said to be nondegenerate if it has no nonzero absolute zero divisors. Similarly, given a Lie algebra $L, x \in L$ is an absolute zero divisor of $L$ if $\operatorname{ad}_{x}^{2}=0$, and $L$ is said to be nondegenerate if it has no nonzero absolute zero divisors.

2.8. Let $B \subseteq V^{+}$be an inner ideal of a Jordan pair $V$. Following [15], the kernel of $B$ is the set $\operatorname{Ker}_{V} B=\left\{y \in V^{-} \mid Q_{B} y=0\right\}$. Then $\left(0, \operatorname{Ker}_{V} B\right)$ is an ideal of the Jordan pair $\left(B, V^{-}\right)$, and the quotient $\operatorname{Sub}_{V} B=\left(B, V^{-}\right) /\left(0, \operatorname{Ker}_{V} B\right)=\left(B, V^{-} / \operatorname{Ker}_{V} B\right)$ is a Jordan pair called the subquotient of $B$. The kernel and the corresponding subquotient of an inner ideal $B \subseteq V^{-}$are defined in a similar way.

The analogues of all these results hold for the abelian inner ideals of a Lie algebra if we replace the Jordan triple product $\{x, y, z\}$ by the left double commutator $[[x, y], z]$ as we describe next. 
2.9. Let $M$ be an abelian inner ideal of a Lie algebra $L$.

(i) The kernel of $M$ is the set $\operatorname{Ker}_{L} M:=\{y \in L \mid[M,[M, y]]=0\}$.

(ii) The pair of $\Phi$-modules $\operatorname{Sub}_{L} M:=\left(M, L / \operatorname{Ker}_{L} M\right)$ with the triple products given by

$$
\begin{aligned}
\{m, \bar{a}, n\} & :=[[m, a], n] \quad \text { for every } m, n \in M \text { and } a \in L, \\
\{\bar{a}, m, \bar{b}\} & :=\overline{[[a, m], b]} \text { for every } m \in M \text { and } a, b \in L,
\end{aligned}
$$

where $\bar{x}$ denotes the coset of $x$ relative to the submodule $\operatorname{Ker}_{L} M$, is a Jordan pair called the subquotient of $M$ [12, Lemma 3.2].

(iii) A $\Phi$-submodule $B$ of $M$ is an inner ideal of $L$ if and only if it is an inner ideal of $\operatorname{Sub}_{L} M$ [12, 3.5(i)].

Definition 2.10. Let $B$ and $C$ be abelian inner ideals of a Lie algebra $L$. We will say that $B$ and $C$ are Jordan-isomorphic $\left(B \cong{ }_{J} C\right)$ if their subquotients $\operatorname{Sub}_{L} B$ and $\mathrm{Sub}_{L} C$ are isomorphic as Jordan pairs.

In 10] a Jordan algebra was attached to any Jordan element of a Lie algebra. Many properties of a Lie algebra are transferred to its Jordan algebras, as well as the fact that the nature of the Jordan element in question is reflected on the structure of the attached Jordan algebra. These facts turn out to be crucial for applications of Jordan theory to Lie algebras.

2.11. Let $a$ be a Jordan element of a Lie algebra $L$. Then $L$ with the new product defined by $x \cdot{ }_{a} y:=\frac{1}{2}[[x, a], y]$ is a nonassociative algebra denoted by $L^{(a)}$, such that

(i) $\operatorname{Ker}_{L} a:=\{x \in L:[a,[a, x]]=0\}$ is an ideal of $L^{(a)}$,

(ii) $L_{a}:=L^{(a)} / \operatorname{Ker}_{L} a$ is a Jordan algebra, called the Jordan algebra of $L$ at $a$.

\section{STANDARD INNER IDEALS}

Throughout this section, and unless otherwise specified, $A$ will be an associative algebra over a ring of scalars $\Phi$ containing $\frac{1}{2}$, and $Z(A)$ will denote the center of $A$. We set $\bar{A}:=A^{-} / Z(A)$ and denote by $x \mapsto \bar{x}$ the canonical homomorphism of Lie algebras of $A^{-}$onto $\bar{A}$.

3.1. Let $V$ be a $\Phi$-submodule of $A$ such that $V V=0$. Then $V$ is an inner ideal of $A^{+}$if and only if it is an (abelian) inner ideal of $A^{-}$: for $u, v \in V$ and $x \in A$, $V V=0$ implies $[[u, x], v]=u x v+v x u=\{u, x, v\}$. An inner ideal $V$ of $A^{-}$such that $V V=0$ will be called a Jordan-Lie inner ideal of $A$.

Definition 3.2. A Lie inner ideal $B$ of $A$ is said to be standard if $B=V+\Omega$, where $V$ is a Jordan-Lie inner ideal of $A$ and $\Omega$ is a $\Phi$-submodule of $Z(A)$.

Notation 3.3. Let $B$ be a Lie inner ideal of $A$. It is clear that $B+Z(A)$ is both a $\Phi$-submodule and a commutative subset of $A$. Denote by $V_{B}$ (or simply by $V$ when there is no risk of confusion) the subset of all zero square elements of $B+Z(A)$.

Lemma 3.4. Let $A$ be semiprime and let $B$ be a Lie inner ideal of $A$.

(i) If $B \subseteq V_{B}+Z(A)$, then $V_{B}$ is a Jordan-Lie inner ideal of $A$, the sum $V_{B}+\bar{Z}(A)$ is direct, and $\left[\left[V_{B}, A\right], V_{B}\right]=\left\{V_{B}, A, V_{B}\right\} \subseteq B$.

(ii) If in addition $V_{B} \subseteq B$ (in particular, if $\left.Z(A) \subseteq B\right)$, then $B=V_{B} \oplus(B \cap$ $Z(A))$ is standard. 
Proof. (i) Set $V:=V_{B}$. It is clear that $V+V \subseteq B+Z(A) \subseteq V+Z(A)$, which implies $V+V \subseteq V$ since $Z(A)$ does not contain any nonzero nilpotent element because $A$ is semiprime. Then, for any $u, v \in V$, we have $0=(u+v)^{2}=2 u v$, and hence $\{u, x, v\}=u x v+v x u=[[u, x], v] \in B$ with $\{u, x, v\}^{2}=0$. This proves that $V$ has the required properties.

(ii) Suppose in addition that $V \subseteq B$. Then the Modular Law applied to the inclusion $B \subseteq V \oplus Z(A)$ yields $B=V \oplus(Z(A) \cap B)$. Finally, $Z(A) \subseteq B$ implies $V \subseteq B+Z(\bar{A}) \subseteq B$, which completes the proof.

Theorem 3.5. Let $A$ be semiprime and let $B$ be a Lie inner ideal of $A$. Then $B$ is standard if and only if the following condition holds:

$$
V_{B} \subseteq B \subseteq V_{B}+Z(A)
$$

Proof. By Lemma 3.4, condition (ST) is sufficient for $B$ to be standard. Suppose then that $B=V \oplus \Omega$ is standard. Clearly, $V \subseteq V_{B}$, and $V_{B} \subseteq B+Z(A) \subseteq V \oplus Z(A)$ implies $V_{B}=V$, which proves that $B$ satisfies (ST).

Proposition 3.6. Let $A$ be semiprime.

(i) If $b$ is an element of $A$ such that $\operatorname{ad}_{b}^{n} A \subseteq Z(A)$, then $\operatorname{ad}_{b}^{n} A=0$ if $n \geq 1$, and $n\left(\operatorname{ad}_{b}^{n-1} A\right)\left(\operatorname{ad}_{b}^{n-1} A\right)=0$ if $n \geq 2$,

(ii) $\bar{A}$ is nondegenerate.

Suppose now that $\frac{1}{6} \in \Phi$. Then:

(iii) Every principal inner ideal $B$ of $A^{-}$is a Jordan-Lie inner ideal.

(iv) If $\bar{b}$ is von Neumann regular in $\bar{A}$, then $b=v+z$, where $v \in[[b, A], b]$, $v^{2}=0, v$ is von Neumann regular in $A$ and $z \in Z(A)$.

Proof. (i) Let $n$ be a positive integer. Since $\mathrm{ad}_{b}$ is a derivation of the associative algebra $A$, we have by the Leibniz rule that $\operatorname{ad}_{b}^{n}(x b)=\operatorname{ad}_{b}^{n}(x) b \in Z(A)$ for all $x \in A$. Hence, by the Jacobi identity, $0=\left[\operatorname{ad}_{b}^{n}(x) b, y\right]=\operatorname{ad}_{b}^{n}(x)[b, y]$ for all $y \in A$. Taking $y=\operatorname{ad}_{b}^{n-1} x$, we obtain $\operatorname{ad}_{b}^{n}(x)^{2}=0$, which implies that $\operatorname{ad}_{b}^{n}(x)=0$ for all $x \in A$, since $\operatorname{ad}_{b}^{n} A \subseteq Z(A)$ and semiprime algebras do not contain nonzero nilpotent central elements. If $n \geq 2$, again by the Leibniz rule, we have $0=\operatorname{ad}_{b}^{n}\left(x_{\operatorname{ad}_{b}^{n-2}}^{n-2}\right)=$ $n\left(\operatorname{ad}_{b}^{n-1} x\right)\left(\operatorname{ad}_{b}^{n-1} y\right)$, as required.

(ii) Let $a \in A$ be such that $\operatorname{ad}_{a}^{2} A \subseteq Z(A)$. By (i), $\operatorname{ad}_{a}^{2} A=0$, and hence, for all $x, y \in A, 0=\operatorname{ad}_{a}^{2}(x y)=2 \operatorname{ad}_{a}(x) \operatorname{ad}_{a}(y)$, which implies $\operatorname{ad}_{a}(x) \operatorname{ad}_{a}(y)=0$. Then $\operatorname{ad}_{a}(x y) \operatorname{ad}_{a}(x)=\operatorname{ad}_{a}(x) y \operatorname{ad}_{a}(x)=0$ implies by semiprimeness of $A$ that $\operatorname{ad}_{a} x=0$ for all $x \in A$, i.e., $a \in Z(A)$, which proves that $\bar{A}$ is nondegenerate.

(iii) Let $B$ be a principal inner ideal of $A^{-}$, i.e., $B=\operatorname{ad}_{x}^{2} A$, where $x$ is a Jordan element of $A^{-}$. It follows from Proposition 2.4(i) and (i) that $B$ is a Jordan-Lie inner ideal of $A$.

(iv) Von Neumann regularity of $\bar{b}$ in $\bar{A}$ means that $\bar{b}$ is a Jordan element of $\bar{A}$ and that there exist $x \in A$ and $z \in Z(A)$ such that $b=[[b, x], b]+z$. By (i), $\operatorname{ad}_{b}^{3} A \subseteq Z(A)$ implies $\operatorname{ad}_{b}^{3} A=0$ and $3\left(\operatorname{ad}_{b}^{2} A\right)\left(\operatorname{ad}_{b}^{2} A\right)=0$. Since we are assuming that $\frac{1}{6} \in \Phi,[[b, x], b]^{2}=0$. The von Neumann regularity of $v=[[b, x], b]$ (in the usual associative sense) is proved as follows: by [10, Lemma 3.2(ii)], the Jordan algebras $A_{v}^{+}$(the $v$-homotope of $A^{+}$) and $\bar{A}_{\bar{v}}=\bar{A}_{\bar{b}}$ are isomorphic. Since the latter 
is unital by [10, Proposition 2.15(ii)], $A_{v}^{+}$is unital, but this implies that $v$ is von Neumann regular in the associative sense: let $\bar{u}$ be the unit element of $A_{v}^{+}$. For any $x \in A$, we have

$$
(v-v u v) x(v-v u v)=v(x-u v x-x v u+u v x v u) v=0,
$$

which implies $v=v u v$ because $A$ is semiprime.

Theorem 3.7. Suppose that $A$ is semiprime and that $\frac{1}{6} \in \Phi$. If $B$ is a Lie inner ideal of $A$ such that every element $\bar{b}$ of $\bar{B}$ is von Neumann regular, then $B$ is standard.

Proof. It follows from Proposition 3.6 (iv) and Theorem 3.5

Definition 3.8. Let $L$ be a Lie algebra and $V$ a Jordan pair.

(i) The length of an inner ideal $B$ of $L$ (respectively $V$ ) is defined as the supremum of the lengths of the chains $0 \subset B_{1} \subset B_{2} \subset \cdots \subset B_{n}$ of inner ideals of $L$ (respectively $V$ ) contained in $B$.

(ii) $L$ (respectively $V$ ) is said to be Artinian if it satisfies the descending chain condition on all inner ideals.

Corollary 3.9. Suppose that $A$ is semiprime and that $\frac{1}{6} \in \Phi$. If $B$ is a Lie inner ideal of $A$ such that $\bar{B}$ has finite length, then $B=\Omega \oplus V$, where $\Omega$ is a $\Phi$-submodule of $Z(A)$ of finite length and $V$ is a Jordan-Lie inner ideal of $A$ of finite length.

Proof. By Proposition 3.6(ii) the Lie algebra $\bar{A}$ is nondegenerate, and since $\bar{B}$ has finite length, we have by [12, Proposition 3.5(iii),(v)] that $\operatorname{Sub}_{\bar{A}} \bar{B}$ is a nondegenerate Artinian Jordan pair, so it is von Neumann regular by [14, Theorem 10.17], which clearly implies that $\bar{B}$ is von Neumann regular in $\bar{A}$. Then it follows from Theorem 3.7 that $B=V \oplus \Omega$ is standard. Since $V \cap Z(A)=0$, any $n$-length chain $0 \subset U_{1} \subset U_{2} \subset \cdots \subset U_{n}$ of Jordan inner ideals of $A$ contained in $V$ gives rise to the $n$-length $\bar{U}_{1} \subset \bar{U}_{2} \subset \cdots \subset \bar{U}_{n}$ of abelian inner ideals of $\bar{A}$ contained in $\bar{B}$, so $V$ has finite length. Similarly, $\Omega$ has finite length as a $\Phi$-module.

\section{Nonstandard INNER IDEAlS}

Throughout this section $A$ will denote a unital semiprime associative algebra $A$ over a field $\Phi$ of characteristic not 2 such that $Z(A)=\Phi 1$. We show a way of constructing Lie inner ideals of $A$ that are not standard.

Theorem 4.1. Let $V$ be a Jordan-Lie inner ideal of $A$, let $V_{0}$ be a hyperplane of $V$ such that $[V,[V, A]] \subseteq V_{0}$, and let $f$ be a functional of $V$ such that $\operatorname{Ker} f=V_{0}$. Then the set $\operatorname{Inn}\left(V, V_{0}, f\right)=\{v+f(v) 1: v \in V\}$ is a Lie inner ideal of $A$ that is not standard.

Proof. To simplify notation, set $B:=\operatorname{Inn}\left(V, V_{0}, f\right)$.

(1) $B$ is a Lie inner ideal. Indeed,

$$
[B,[B, A]]=[V,[V, A]] \subseteq V_{0}=\operatorname{Ker}(f) \subseteq B
$$

and

$$
[B, B] \subseteq[V, V] \subseteq V V=0 .
$$

(2) $B \cap Z(A)=B \cap \Phi 1=0$, since $v+f(v) 1=\alpha 1$ implies $v=(f(v)-\alpha) 1$, and hence $v=0$ and $\alpha=f(v)=0$. 
(3) By definition of $B, V \subseteq B \oplus \Phi 1$, so $V \subseteq V_{B}$ since $V V=0$. Conversely, let $b+\alpha 1 \in V_{B}$, with $b=v+f(v) 1$. Then

$$
0=(b+\alpha 1)^{2}=(v+(f(v)+\alpha) 1)^{2}=2(f(v)+\alpha) v+(f(v)+\alpha)^{2} 1
$$

implies $\alpha+f(v)=0$, so $b+\alpha 1=v \in V$, which proves that $V_{B} \subseteq V$.

(4) Since $V \cap B=\operatorname{Ker}(f)=V_{0}$, with $V_{0}$ a hyperplane of $V$, we have that $V_{B}=V$ is not contained in $B$. So $B$ is not standard by Theorem 3.5 .

Corollary 4.2. Any zero square element $x \in A$ which is not von Neumann regular produces the nonstandard inner ideal $\operatorname{Inn}(\Phi x \oplus x A x, x A x, f), f(x A x)=0, f(x)=1$.

We end this section by proving that $\operatorname{Inn}\left(V, V_{0}, f\right)$ is independent up to Jordan isomorphism of the choice of the functional $f, \operatorname{Ker} f=V_{0}$.

Proposition 4.3. Let $V$ be a Jordan-Lie inner ideal of $A$, and let $V_{0}$ be a hyperplane of $V$ such that $[V,[V, A]] \subseteq V_{0}$. If $f, g$ are functionals of $V$ such that $\operatorname{Ker} f=$ $\operatorname{Ker} g=V_{0}$, then the Lie inner ideals $B=\operatorname{Inn}\left(V, V_{0}, f\right)$ and $C=\operatorname{Inn}\left(V, V_{0}, g\right)$ are Jordan-isomorphic.

Proof. Note first that $\operatorname{Ker}_{A^{-}} B=\operatorname{Ker}_{A^{-}} C=\operatorname{Ker}_{(A, A)} V$. Now we have that the pair of linear mappings $(\varphi, \mathrm{Id})$, where $\varphi: B \rightarrow C$ is defined by $\varphi(v+f(v))=v+g(v)$, $v \in V$, and Id is the identity mapping on the vector space $A / \operatorname{Ker}_{A^{-}} B$, is a Jordan pair isomorphism of $\operatorname{Sub}_{A^{-}} \operatorname{Inn}\left(V, V_{0}, f\right)$ onto $\operatorname{Sub}_{A^{-}} \operatorname{Inn}\left(V, V_{0}, g\right)$.

\section{LIE INNER IDEALS OF CENTRALLY CLOSED PRIME ALGEBRAS}

Throughout this section $A$ will denote a centrally closed prime associative algebra over a field $\Phi$ (of characteristic not 2 or 3 ); i.e., $A$ is prime and its extended centroid (see [1 for a definition) is $\Phi$ itself. Clearly, a simple associative algebra is centrally closed over its centroid.

The following lemma, which was proved in [2, Lemma 3.10] for simple associative algebras, is a refinement of a more general result by Martindale and Miers for prime algebras and is the key tool to determine the Lie inner ideals of a centrally closed prime algebra.

Lemma 5.1. Let $a \in A$ be a Jordan element of $A^{-}$. Then there exists a central element $z$ in $A$, necessarily unique, such that $(a-z)^{2}=0$.

Proof. By [16, Corollary 1], there exists $\lambda \in \Phi$ such that $(a-\lambda 1)^{2}=0$, the formula making sense in the unital hull $\hat{A}=A+\Phi 1$ of $A$. If $A$ is unital, then $\lambda 1 \in Z(A)$. If $A$ is nonunital, then $a^{2}-2 \lambda a=-\lambda^{2} 1$ implies $\lambda=0$.

Proposition 5.2. Let $B$ be a Lie inner ideal of $A$. In any of the following situations $B$ is standard:

(i) A is nonunital.

(ii) Every zero square element of $A$ is von Neumann regular.

(iii) $Z(A) \subseteq B$.

(iv) $B$ is a maximal Lie inner ideal.

In case (i), $B=V_{B}$ is in fact a Jordan-Lie inner ideal.

Proof. (i) Since $A$ is nonunital, $Z(A)=0$. Hence $V_{B} \subseteq B$ (by definition) and $B \subseteq V_{B}$ by Lemma 5.1. Now we have by Lemma 3.4(i) that $B=V_{B}$ is a JordanLie inner ideal. 
(ii) By Lemma 5.1, $B \subseteq V_{B}+Z(A)$. Hence $V_{B}$ is a Jordan-Lie inner of $A$ such that $\left\{V_{B}, A, V_{B}\right\} \subseteq B$ by Lemma 3.4(i). Since every zero square element of $A$ is von Neumann regular, $V_{B}=\left\{V_{B}, A, V_{B}\right\}=\left[\left[V_{B}, A\right], V_{B}\right] \subseteq B$, which implies that $B$ is standard by Lemma 3.4(ii).

(iii) The inclusion $Z(A) \subseteq B$ implies $V_{B} \subseteq B$. Then the proof follows as in (i).

(iv) It follows from (iii) since $B+Z(A)$ is a Lie inner ideal.

Corollary 5.3. Let $X$ be a right vector space over a division ring $\Delta$ of characteristic not 2 or 3 . Then every Lie inner ideal of $\operatorname{End}_{\Delta}(X)$ (regarded as a $Z(\Delta)$-algebra) is standard.

Proof. The ring $\operatorname{End}_{\Delta}(X)$ is prime (in fact, primitive) and von Neumann regular. Moreover, it is centrally closed over $Z(\Delta)$ by [1, Theorem 4.3.7(ix)]. Thus we can apply Proposition 5.2 (ii) to get that any Lie inner ideal of $\operatorname{End}_{\Delta}(X)$ is standard.

Theorem 5.4. Let $B$ be a Lie inner ideal of $A$. Then either

(i) $B=V$, where $V$ is a Jordan-Lie inner ideal of $A$,

(ii) $B=V \oplus \Phi 1$, where $V$ is as in (i), or

(iii) $B=\operatorname{Inn}\left(V, V_{0}, f\right)$ is a nonstandard inner ideal.

Note that in cases (ii) and (iii), A is necessarily unital.

Proof. If $A$ is nonunital, then every Lie inner ideal of $A$ is a Jordan-Lie inner ideal by Proposition 5.2(i). Suppose that $A$ has a unit and therefore $Z(A)=\Phi 1$. If $1 \in B$, then $Z(A)=\Phi 1$ is contained in $B$, so we have by Proposition 5.2 (iii) that $B=$ $V \oplus \Phi 1$ is standard. Suppose then that $1 \notin B$. Then, again by Proposition 5.2 (iii), $B \oplus \Phi 1=V \oplus \Phi 1$ for some Jordan-Lie inner ideal $V$ of $A$. We also have that if $v+\alpha 1$ and $v+\beta 1$ belong to $B$, then $\alpha=\beta$ (otherwise 1 would belong to $B$, which has been discarded). Thus there exists $f \in V^{*}$ such that $B=\{v+f(v) 1: v \in V\}$. If $f=0$, then $B=V$ and we have case (i). Suppose then that $f$ is nonzero and let $u \in V$ be such that $f(u)=1$. Then $V=\Phi u \oplus V_{0}$, with $V_{0}=\operatorname{Ker} f$. Clearly, $V \cap B=V_{0}$, and since $[V,[V, A]]=[B,[B, A]]$, we have $[V,[V, A]] \subseteq V \cap B=V_{0}$. Thus $B=\operatorname{Inn}\left(V, V_{0}, f\right)$ is nonstandard, which completes the proof.

Corollary 5.5. If $A$ is nonunital, then every Lie inner ideal of $A$ is standard. If $A$ is unital, then the following conditions are equivalent:

(i) Every zero square element of $A$ is von Neumann regular.

(ii) Every Lie inner ideal of $A$ is standard.

Proof. It follows from Theorem 5.4 together with Corollary 4.2.

We give a primitive algebra which is not centrally closed but with the property that all its Lie inner ideals are standard.

Example 5.6. Let $X$ be an infinite-dimensional complex vector space, let $\mathcal{F}(X)$ be the simple complex associative algebra of all finite rank operators on $X$, and set $A=\mathcal{F}(X) \oplus \mathbb{R} \operatorname{Id}_{X}$. Then $A$ is a real primitive algebra which is not centrally closed, its extended centroid being the complex field. However, every Lie inner ideal $B$ of $A$ is standard. In fact, either $B=V$, where $V$ is a Jordan-Lie inner ideal, or $B=\mathbb{R I d}_{X} \oplus V$. 


\section{LIE INNER IDEALS OF SEMIPRIME ALGEBRAS WITH FINITENESS CONDITIONS}

In this last section we see that if $A$ is a semiprime algebra coinciding with its socle, then every Lie inner ideal of $A$ is standard. Further, we describe the Lie inner ideal structure of these algebras in associative terms.

Theorem 6.1. Let $A$ be a semiprime associative algebra over a ring of scalars $\Phi$ containing $\frac{1}{6}$ and let $B$ be a Lie inner ideal of $A$.

(i) If $A$ coincides with its socle, then $B=\Omega \oplus R L$, where $\Omega$ is a $\Phi$-submodule of $Z(A), R$ is a right ideal of $A$, and $L$ is a left ideal of $A$ with $L R=0$ and $R L=R \cap L$.

(ii) If $A$ is actually Artinian, then $B=\Omega \oplus e A f$, where $\Omega$ is a $\Phi$-submodule of $Z(A)$ and $e, f$ are idempotents of $A$ such that $f e=0$.

Proof. (i) By the structure of the socle, $A=\bigoplus M_{i}$ is a direct sum of minimal ideals, each of which is a simple (and therefore centrally closed over its centroid) algebra of characteristic not 2 or 3 coinciding with its socle. Let $b \in B$. Then $b=\sum b_{i}$, where each $b_{i} \in M_{i}$ and $b_{i}=0$ up to a finite subset of indexes. By Lemma 5.1, for any $b_{i}$ there exists a unique $z_{i} \in Z\left(M_{i}\right)$ such that $\left(b_{i}-z_{i}\right)^{2}=0$. Set $z=\sum z_{i}$. Then $z \in Z(A)$ and $(b-z)^{2}=0$, which proves that $B$ satisfies condition (i) of Lemma 3.4. Moreover, since $A$ is von Neumann regular (because it coincides with its socle), $B$ also satisfies condition (ii) of Lemma 3.4, and so $B=V \oplus(B \cap Z(A))$ is standard. Again using the von Neumann regularity of $A^{+}$, we get that $V=\bigoplus V_{i}$, where each $V_{i}$ is a Jordan-Lie inner ideal of $M_{i}$. By [13, Theorem 3(ii)], for each index $i, V_{i}=R_{i} L_{i}$, where $R_{i}$ is a right ideal of $M_{i}$ and $L_{i}$ is a left ideal. Then $V=R L$, where $R=\bigoplus R_{i}$ and $L=\bigoplus L_{i}$, with $L R=0$ since $V V=0$. The equality $R L=R \cap L$ follows because $A$ is von Neumann regular.

(ii) If $A$ is Artinian, then it coincides with its socle. Hence, by (i), $B=R L \oplus(B \cap$ $Z(A))$ is standard. Now we can apply the structure of one-sided ideals of semiprime Artinian rings to get that $R=e A$ and $L=A f$, where $e, f$ are idempotents of $A$ with $f e=0$, or use [17, Theorem 1] to get $V=e A f$ directly.

\section{ACKNOWLEDGMENTS}

The author thanks the referee for many constructive remarks, Esther García and Miguel Gómez Lozano for a preliminary version of Proposition 3.6. and Jose Brox for his careful reading of the manuscript.

\section{REFERENCES}

[1] K.I. Beidar, W.S. Martindale and A.V. Mikhalev, Rings with Generalized Identities. Pure and Applied Mathematics, 196, Marcel Dekker, Inc., New York, 1996. MR1368853 (97g:16035)

[2] Georgia Benkart, The Lie inner ideal structure of associative rings, J. Algebra 43 (1976), no. 2, 561-584. MR0435149 (55 \#8110)

[3] Georgia Benkart, On inner ideals and ad-nilpotent elements of Lie algebras, Trans. Amer. Math. Soc. 232 (1977), 61-81. MR0466242 (57 \#6122)

[4] Arjeh M. Cohen, Gábor Ivanyos, and Dan Roozemond, Simple Lie algebras having extremal elements, Indag. Math. (N.S.) 19 (2008), no. 2, 177-188, DOI 10.1016/S0019-3577(09)000032. MR2489325(2010d:17012)

[5] Cristina Draper, Antonio Fernández López, Esther García, and Miguel Gómez Lozano, The socle of a nondegenerate Lie algebra, J. Algebra 319 (2008), no. 6, 2372-2394, DOI 10.1016/j.jalgebra.2007.10.042. MR2388311(2010a:17045) 
[6] C. Draper, A. Fernández López, E. García, and M. Gómez Lozano, The inner ideals of the simple finite dimensional Lie algebras, J. Lie Theory 22 (2012), no 4, 970-929. MR3052677

[7] John R. Faulkner, On the geometry of inner ideals, J. Algebra 26 (1973), 1-9. MR0367002 (51 \#3247)

[8] Antonio Fernández López, Esther García, and Miguel Gómez Lozano, The Jordan socle and finitary Lie algebras, J. Algebra 280 (2004), no. 2, 635-654, DOI 10.1016/j.jalgebra.2004.06.013. MR2089256(2005i:17007)

[9] Antonio Fernández López, Esther García, and Miguel Gómez Lozano, Inner ideals of finitary simple Lie algebras, J. Lie Theory 16 (2006), no. 1, 97-114. MR2196417 (2006m:17020)

[10] Antonio Fernández López, Esther García, and Miguel Gómez Lozano, The Jordan algebras of a Lie algebra, J. Algebra 308 (2007), no. 1, 164-177, DOI 10.1016/j.jalgebra.2006.02.035. MR2290916 (2007k:17049)

[11] Antonio Fernández López, Esther García, and Miguel Gómez Lozano, An Artinian theory for Lie algebras, J. Algebra 319 (2008), no. 3, 938-951, DOI 10.1016/j.jalgebra.2007.10.038. MR2379087 (2010a:17046)

[12] A. Fernández López, E. García, M. Gómez Lozano, and E. Neher, A construction of gradings of Lie algebras, Int. Math. Res. Not. 2007, no. 16, Art. ID rnm051, 34 pp. MR2353091 (2008j:17057)

[13] Antonio Fernández López and Eulalia García Rus, Inner ideals in quadratic Jordan algebras of infinite capacity, Int. J. Math. Game Theory Algebra 9 (1999), no. 1, 35-54. MR.1697472 (2000f:17044)

[14] Ottmar Loos, Jordan pairs, Lecture Notes in Mathematics, Vol. 460, Springer-Verlag, Berlin, 1975. MR0444721(56 \#3071)

[15] Ottmar Loos and Erhard Neher, Complementation of inner ideals in Jordan pairs, J. Algebra 166 (1994), no. 2, 255-295, DOI 10.1006/jabr.1994.1151. MR.1279257 (95f:17028)

[16] W. S. Martindale III and C. Robert Miers, On the iterates of derivations of prime rings, Pacific J. Math. 104 (1983), no. 1, 179-190. MR683736 (84c:16033)

[17] Kevin McCrimmon, Inner ideals in quadratic Jordan algebras, Trans. Amer. Math. Soc. 159 (1971), 445-468. MR0279145 (43 \#4871)

[18] A. A. Premet, Lie algebras without strong degeneration, Mat. Sb. (N.S.) 129(171) (1986), no. 1, 140-153 (Russian). MR830100 (87g:17018)

[19] A. A. Premet, Inner ideals in modular Lie algebras, Vestsī Akad. Navuk BSSR Ser. Fīz.-Mat. Navuk 5 (1986), 11-15, 123 (Russian, with English summary). MR876665 (88d:17013)

[20] Alexander Premet and Helmut Strade, Simple Lie algebras of small characteristic. I. Sandwich elements, J. Algebra 189 (1997), no. 2, 419-480, DOI 10.1006/jabr.1996.6861. MR $1438184(98 \mathrm{e}: 17029)$

[21] Oleg N. Smirnov, Simple associative algebras with finite Z-grading, J. Algebra 196 (1997), no. 1, 171-184, DOI 10.1006/jabr.1997.7087. MR1474168 (98i:16045)

[22] Oleg N. Smirnov, Finite Z-gradings of Lie algebras and symplectic involutions, J. Algebra 218 (1999), no. 1, 246-275, DOI 10.1006/jabr.1999.7880. MR.1704686 (2000f:17034)

[23] E. Zelmanov, Lie algebras with finite grading, Math. USSR Sbornik 52 (1985), 347-385 (English translation). MR.0752226 (86d:17016)

Departamento de Álgebra, Geometría y Topología, Universidad de Málaga, 29071, MÁlaga, SPAIN

E-mail address: emalfer@uma.es 